Article

\title{
Theoretical Analysis of Effective Thermal Conductivity for the Chinese HTR-PM Heat Transfer Test Facility
}

\author{
Cheng Ren *, Xingtuan Yang, Haijun Jia, Yueyuan Jiang and Wei Xiong \\ Key Laboratory of Advanced Reactor Engineering and Safety of Ministry of Education, Collaborative Innovation \\ Center of Advanced Nuclear Energy Technology, Institute of Nuclear and New Energy Technology, \\ Tsinghua University, Beijing 100084, China; yangxt107@sina.com (X.Y.); jiaha@tsinghua.edu.cn (H.J.); \\ jiangyy@sina.com (Y.J.); xiongw@sina.com (W.X.) \\ * Correspondence: rcheng@mail.tsinghua.edu.cn; Tel.: +86-138-1010-7661
}

Academic Editor: Yuyuan Zhao

Received: 26 September 2016; Accepted: 9 January 2017; Published: 12 January 2017

\begin{abstract}
The Chinese high temperature gas-cooled reactor pebble bed module (HTR-PM) demonstration project has attracted increasing attention. In order to support the project, a large-scale heat transfer test facility has been constructed for pebble bed effective thermal conductivity measurement over the whole temperature range $\left(0 \sim 1600{ }^{\circ} \mathrm{C}\right)$. Based on different heat transfer mechanisms in the randomly packed pebble bed, three different types of effective thermal conductivity have been theoretically evaluated. A prediction of the total effective thermal conductivity of the pebble bed over the whole temperature range is provided for the optimization of the test facility and guidance of further experiments.
\end{abstract}

Keywords: high temperature gas-cooled reactor pebble bed module; heat transfer test facility; effective thermal conductivity

\section{Introduction}

Increasing attention has been paid to the high temperature helium-cooled pebble bed reactor (HTR) due to its high efficiency, high levels of passive safety, and potential usage for hydrogen production [1,2]. The Chinese high temperature gas-cooled reactor pebble bed module (HTR-PM) demonstration project, oriented by the Institute of Nuclear and New Energy Technology of Tsinghua University (INET), has been installed at the Shidaowan plant in Shandong Province, China and is scheduled to go online in 2017 [3,4].

HTR-PM has a cylindrical pebble bed core with a diameter of $3 \mathrm{~m}$ and a height of $11 \mathrm{~m}$, and thousands of spherical fuel elements are randomly packed inside. In engineering practice, values of the effective thermal conductivity of the pebble bed core of HTR-PM at different temperatures are essential parameters required in safety analysis and thermal calculation of the reactor [5]. The effective thermal conductivity has been closely related to the HTR safe characteristic. When a loss-of-coolant accident happens, the residual heat must be removed from the core in time, which directly depends on the effective thermal conductivity of the pebble bed [6-9]. A relevant experiment named SANA-1 [1-12] designed by the Research Center Juelich has historically been carried out for validation of the afterheat removal capacity of HTR. The size of the pebble bed and the highest test temperature (below $1000{ }^{\circ} \mathrm{C}$ ) in SANA-1 were limited. The temperature range of SANA-1 was unable to cover the whole temperature range of the safety analysis of HTR-PM $\left(0 \sim 1600^{\circ} \mathrm{C}\right)$.

In order to support the HTR-PM project, Tsinghua University has designed and constructed a full-scale heat transfer test facility for pebble bed equivalent conductivity measurement (TF-PBEC) over the whole temperature range $\left(0 \sim 1600{ }^{\circ} \mathrm{C}\right)[13,14]$, as shown in Figure 1 . The function of the TF-PBEC is to create a full-size pebble bed to simulate the complicated thermal transfer condition 
in the real reactor core of HTR-PM. The TF-PBEC is an integrated experimental system and has the structure of the internal heating type resistance furnace. About 70,000 machined graphite spheres with a diameter of $60 \mathrm{~mm}$ were randomly packed in an annular test zone bounded by inner and outer walls inside the TF-PBEC to simulate the fuel packing structure, as shown in Figure 1 [5]. The radius of the inner wall of the pebble bed is $500 \mathrm{~mm}$ and that of the outer wall is set to be $2000 \mathrm{~mm}$. The height of the pebble bed is set to be $1000 \mathrm{~mm}$. The temperature distribution in the pebble bed under helium atmosphere conditions will be measured to determine the effective thermal conductivity values of the pebble bed with temperature up to $1600{ }^{\circ} \mathrm{C}$. In order to optimize the design of the facility, a theoretical prediction of the effective thermal conductivity of the pebble bed over the whole temperature range is needed. This paper carries out detailed theoretical analysis on the effective thermal conductivity of the pebble bed in the HTR-PM heat transfer test facility, which is necessary for the design and optimization of the test facility and further experiments. In the SANA experiment, the effective thermal conductivity of a graphite pebble bed at the temperature range of $0 \sim 1000{ }^{\circ} \mathrm{C}$ was measured. Due to the similarity of the two reactors (Chinese HTR-PM and Germany HTR-Module), the graphite pebbles used in the TF-PBEC are similar to those in the SANA experiment. Hence, the experimental data used for analysis in this paper is from SANA's data set.

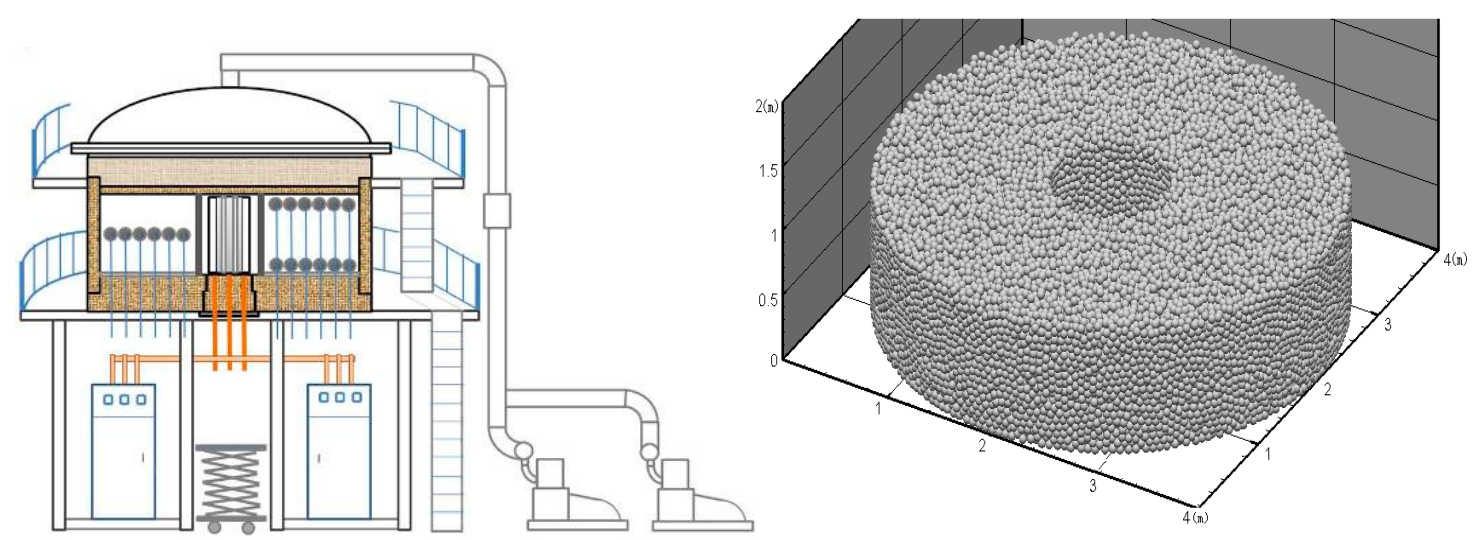

Figure 1. Structure diagram and pebble bed of the test facility for pebble bed equivalent conductivity measurement (TF-PBEC).

\section{Heat Transfer Mechanisms in Pebble Bed}

As above, the effective thermal conductivity of the high temperature pebble bed reactor, which is usually used to simulate the heat transfer in the reactor core under normal operating or severe accident conditions, is derived by integrating all the relevant heat transfer mechanisms in the pebble bed into a single representative conduction process.

More specifically, the pebble bed in the upcoming experiment is a randomly packed bed filled with a stagnant gas. Four different heat transfer mechanisms occur, namely: (1) conduction through solid spheres; (2) conduction through the stagnant gas phase that fills voids in-between spheres; (3) conduction through contact areas between adjacent spheres; and (4) radiation between sphere surfaces, as shown in Figure 2. 


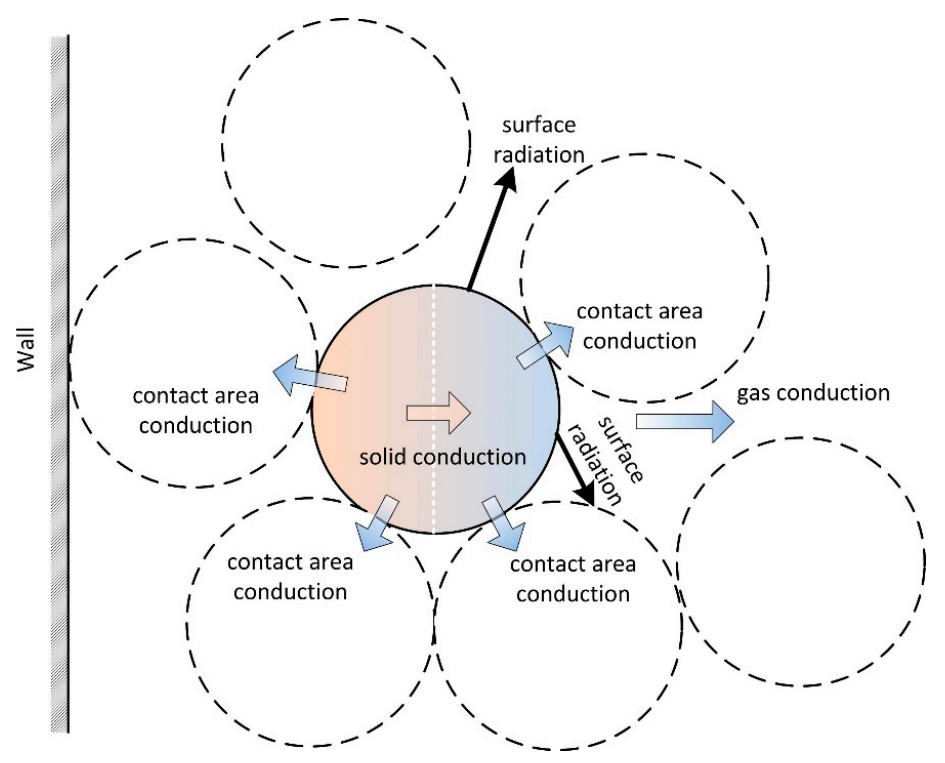

Figure 2. Heat transfer mechanisms in packed pebble bed.

\section{Effective Thermal Conductivity Analysis}

In the pebble bed, the heat flux is to be transported simultaneously along three different paths, namely: (1) solid conduction-surface radiation-solid conduction process; (2) solid conduction-gas conduction-solid conduction process and (3) solid conduction-contact area conduction-solid conduction process. Hence, the total effective conductivity is considered to consist of these three different types of effective conductivity, which must be evaluated separately.

\subsection{Solid Conduction + Surface Radiation + Solid Conduction}

Zehner and Schluender [11] proposed a cell model in 1970, which can be used to describe this type effective thermal conductivity. A unit cell consists of two half spheres with point contact and void between spheres. The pebble bed in the cell model is formed by orderly arrangement of this kind unit cells and heat transfers in the pebble bed by radiation of sphere surfaces and conduction in spheres. Breitbach and Barthels [6] noted the cell model did not consider the radiation from gaps outside the cell and improved the formula. In this paper, we adopt this developed formula, as shown below:

$$
\lambda_{e}^{r}=\left\{\left[1-(1-\varepsilon)^{1 / 2}\right] \varepsilon+\frac{(1-\varepsilon)^{1 / 2}}{2 / \varepsilon_{r}-1} \cdot \frac{B+1}{B} \cdot \frac{1}{1+\frac{1}{\left(2 / \varepsilon_{r}-1\right) \Lambda}}\right\} \cdot 4 \sigma T^{3} d
$$

where $\lambda_{e}^{r}$ is the first type effective thermal conductivity due to the solid conduction-surface radiation-solid conduction heat transfer process; $F_{E}=\left[1-(1-\varepsilon)^{1 / 2}\right] \varepsilon+\frac{(1-\varepsilon)^{1 / 2}}{2 / \varepsilon_{r}-1} \cdot \frac{B+1}{B} \cdot \frac{1}{1+\frac{1}{\left(2 / \varepsilon_{r}-1\right) \Lambda}}$ is defined as the radiation exchange factor; $\varepsilon$ is the porosity of the pebble bed; $\varepsilon_{r}$ is the pebble emissivity; $B$ is the deformation parameter related to the porosity, which was given by Zehner and Schluender as $B=1.25\left(\frac{1-\varepsilon}{\varepsilon}\right)^{10 / 9}$. However, Hsu et al. found that $B=1.364\left(\frac{1-\varepsilon}{\varepsilon^{1.055}}\right)$ led to a more accurate prediction, which is adopted in the following analysis. $\Lambda=\frac{\lambda_{s}}{4 \sigma T^{3} d}$ is the dimensionless solid conductivity. $\sigma$ is the Stephan-Boltzmann constant. $\lambda_{s}$ is the heat conductivity of the pebble, which is temperature dependent. $d$ is the diameter of the pebble.

The first type effective thermal conductivity calculated by Equation (1) is shown in Figure 3, using parameters of the SANA experiment under helium condition, which can be found in [12]. The pebbles used in SANA experiment were also graphite spheres with the same diameter in our experiment. As we can see, this type effective thermal conductivity grows quickly with temperature rising due 
to the radiation effect. Although the heat conduction ability of the pebble itself declines with rising temperature, the whole heat transfer ability of the pebble bed increases quickly due to the sharp enhancing of the surface radiation of the pebbles.

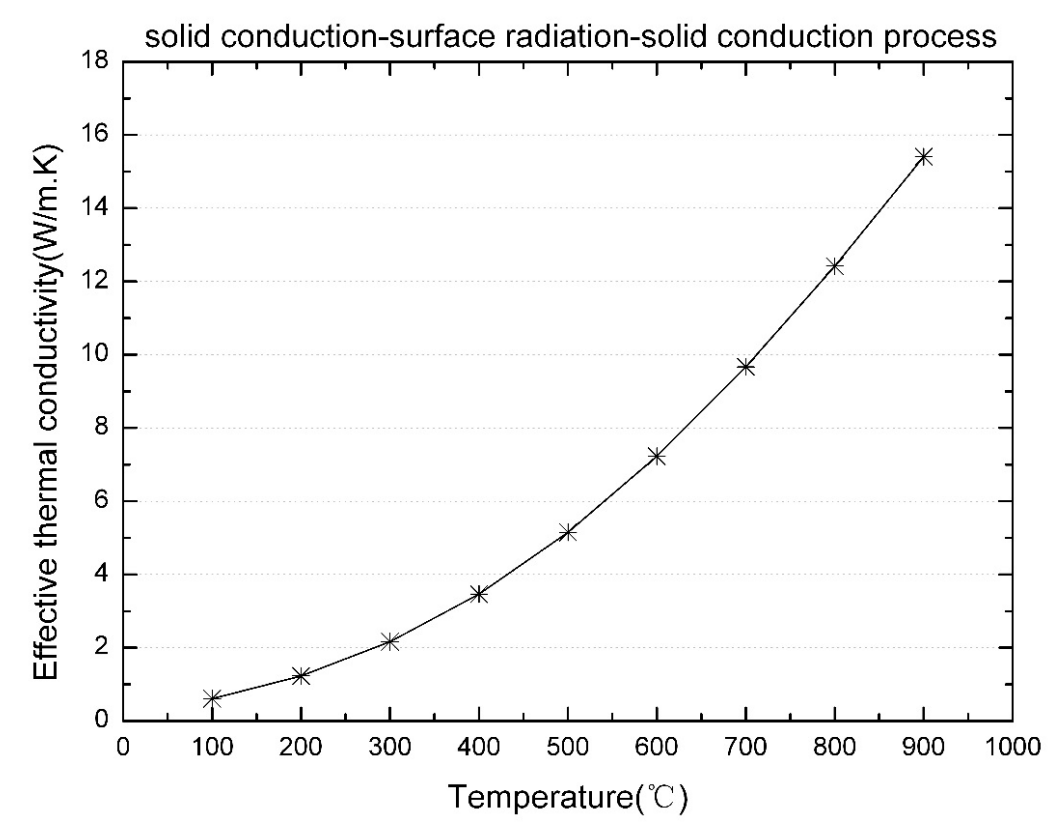

Figure 3. Effective thermal conductivity due to the solid conduction-surface radiation-solid conduction heat transfer process.

\subsection{Solid Conduction + Gas Conduction + Solid Conduction}

In this part, we consider the second type effective thermal conductivity caused by heat transfer through solid spheres and gas phase with stagnant flow. Point contact will be assumed and thermal radiation is neglected.

The packed pebble bed consisting of mono-sized spheres with the presence of a stagnant fluid can be considered as a porous media filled with still working medium. The second type of effective thermal conductivity then can be considered as the prediction of the stagnant thermal conductivity of the porous media. Zehner and Schlunder presented an empirical correlation for the stagnant thermal conductivity [15]. The accuracy of the correlation has been confirmed by tests done by $\mathrm{V}$. Prasad et al. [16]:

$$
\frac{\lambda_{e}^{g}}{\lambda_{f}}=1-\sqrt{1-\varepsilon}+\frac{2 \sqrt{1-\varepsilon}}{1-\lambda B}\left[\frac{(1-\lambda) B}{(1-\lambda B)^{2}} \ln \left(\frac{1}{\lambda B}\right)-\frac{B+1}{2}-\frac{B-1}{1-\lambda B}\right]
$$

where $\lambda_{e}^{g}$ is the second type effective thermal conductivity due to the solid conduction-gas conduction-solid conduction heat transfer process; $\lambda_{f}$ is the heat conductivity of the stagnant gas, which is temperature dependent; $\lambda$ is the ratio of the thermal conductivity of the solid phase and the surrounding fluid matrix.

The second type of effective thermal conductivity calculated by Equation (2) is shown in Figure 4, using parameters of the SANA experiment under helium conditions. As we can see, this type of effective thermal conductivity only has a slight increase with rising temperature. The heat conduction ability of the helium increases with rising temperature, while the heat conduction ability of the graphite spheres declines. Hence, the total heat transfer ability increases slowly. 


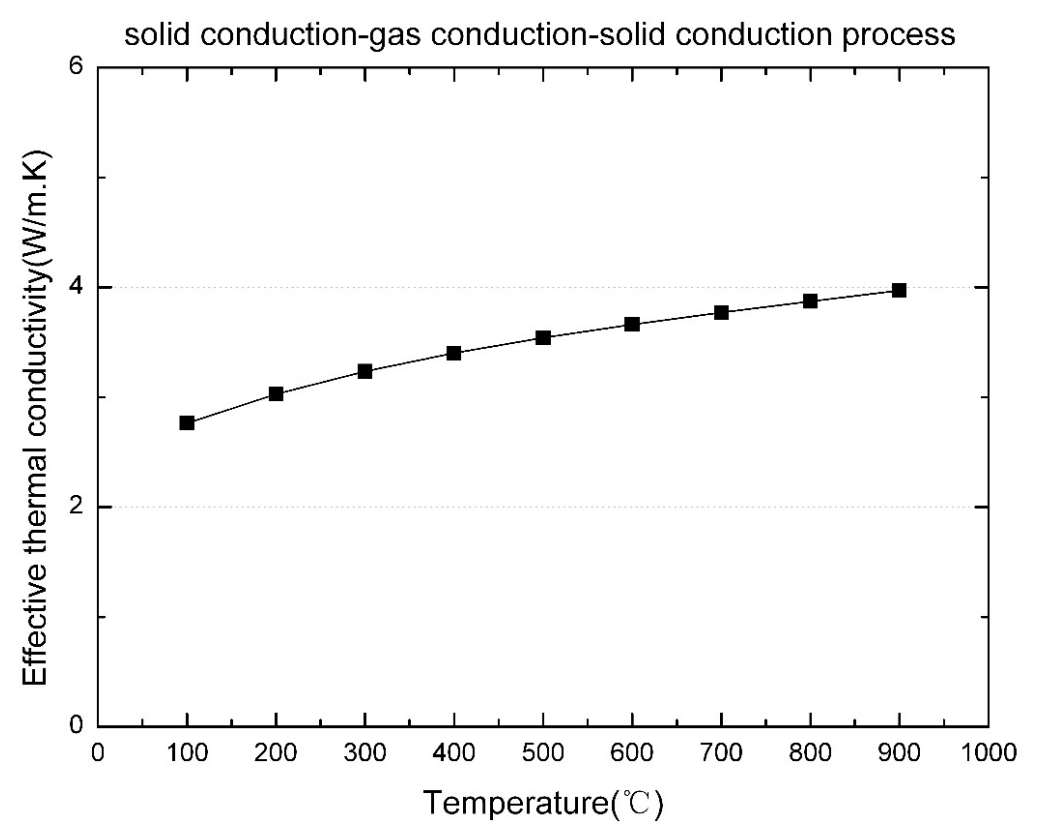

Figure 4. Effective thermal conductivity due to the solid conduction-gas conduction-solid conduction heat transfer process.

\subsection{Solid Conduction + Contact Area Conduction + Solid Conduction}

Contact area is the contact region between two adjacent spheres, usually caused by external pressures acting onto the spheres or their own weight in a packed bed. It is closely related to the elasticity of the sphere's material and usually increases in size with external load. It has been pointed out by many researchers that it is quite important to take the contact area thermal conduction into account at high solid-to-fluid thermal conductivity ratios $\left(\lambda \geq 10^{3}\right)$ [11]. Hence, in this part, we consider the third type of effective thermal conductivity caused by heat transfer through a packed pebble bed with finite contact areas.

The contact area between two adjacent spheres can be calculated by a model presented by Kaviany using Hertzian deformation [17]. The third type of effective thermal conductivity caused by finite contact area conduction can be described by the following correlation:

$$
\frac{\lambda_{e}^{c}}{\lambda_{s}}=\left[\frac{3\left(1-\mu_{p}^{2}\right)}{4 E_{s}} f R\right]^{1 / 3} \cdot \frac{1}{0.531 S}\left(\frac{N_{A}}{N_{L}}\right)
$$

where $\lambda_{e}^{c}$ is the third type effective thermal conductivity due to the solid conduction-contact area conduction-solid conduction heat transfer process; $r_{c}=\left[\frac{3\left(1-\mu_{p}^{2}\right)}{4 E_{s}} f R\right]^{1 / 3}$ is the radius of the contact area between two spheres; $\mu_{p}$ is the Poisson ratio; $E_{s}$ is the Young modules; $f$ is the collinear force acting on the spheres; $R$ is the radius of the graphite sphere; $S$ is the constant related to the structure of packed bed; $N_{A}$ and $N_{L}$ are the number of spheres per unit area and unit length in the packed bed, respectively. Kaviany [17] studied three different close-packing ordered arrangements (simple cubic packing, face-centered cubic packing, and body centered cubic packing) and provided values of the structural parameters. Based on the study, a randomly packed bed can be considered depending on porosity.

The third type effective thermal conductivity calculated by Equation (3) is shown in Figure 5, using parameters of the SANA experiment under helium atmosphere. As we can see, the type effective thermal conductivity shows a slight decline when temperature rises due to the graphite material characteristics. 


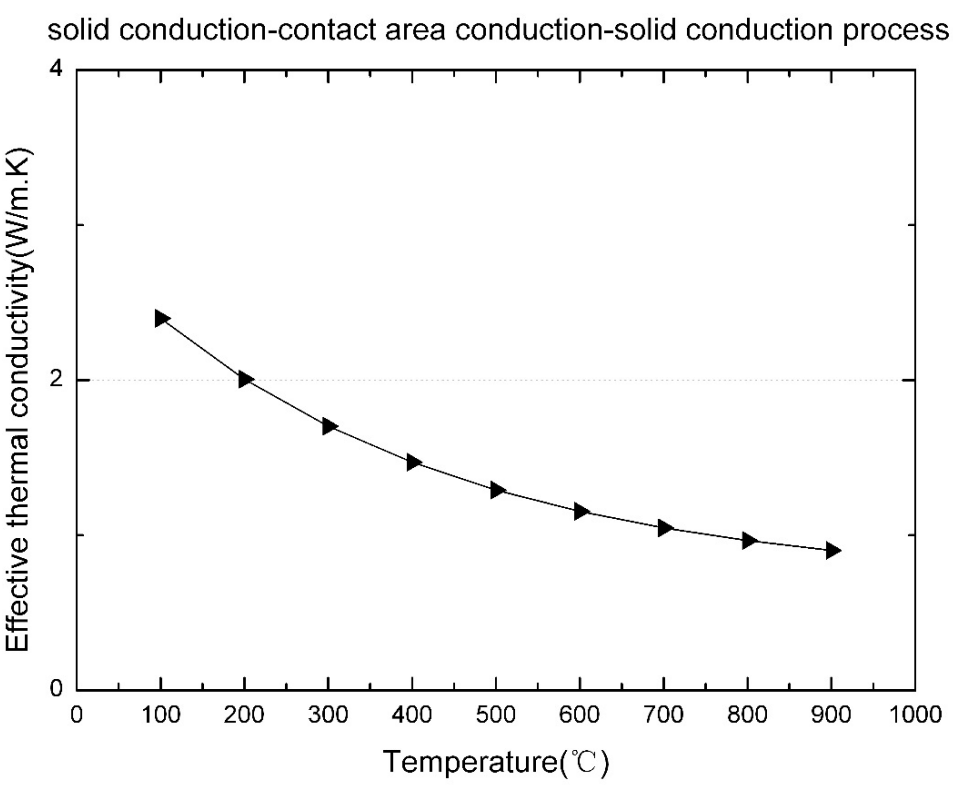

Figure 5. Effective thermal conductivity due to the solid conduction-contact area conduction-solid conduction heat transfer process.

Because there are three heat transfer processes that coexist in the packed bed, the total effective thermal conductivity of the pebble bed can be considered as the summation of the above three types of thermal conductivities:

$$
\lambda_{e}^{t}=\lambda_{e}^{r}+\lambda_{e}^{g}+\lambda_{e}^{c}
$$

the calculated total effective thermal conductivity and the measured values in SANA experiment under helium conditions are shown in Figure 6. As we can see, the calculated effective conductivity and the measured data fit well. Among these three heat transfer mechanisms, heat transfer by radiation plays a dominating role as temperature rises, while heat transfer through gas conduction and contact area conduction contributes to the major portion in the lower temperature zone.

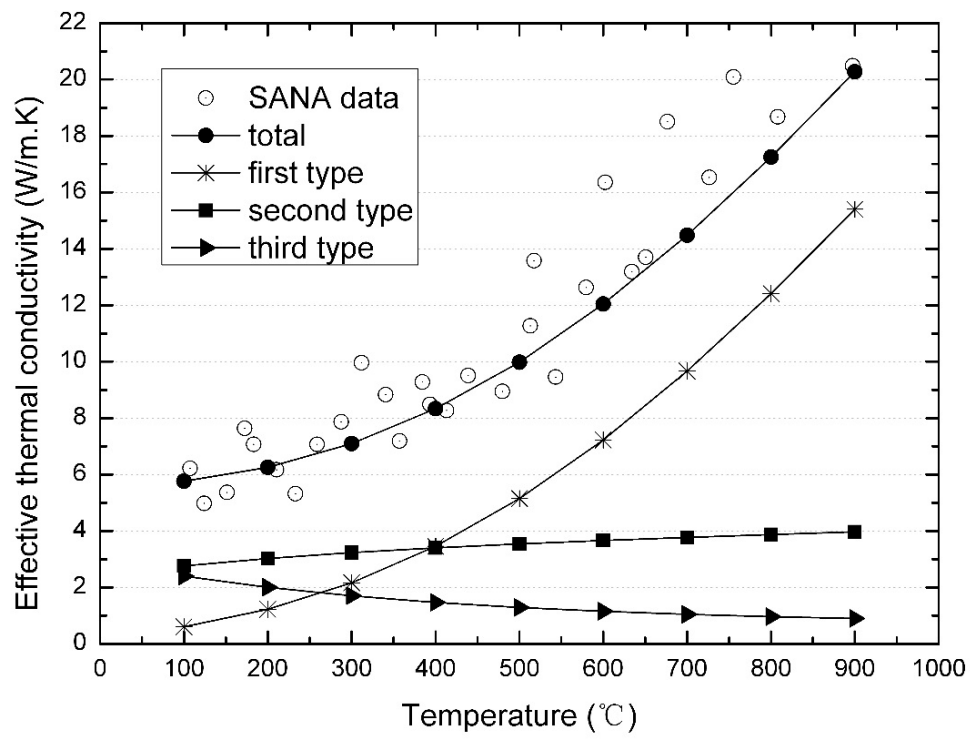

Figure 6. Total effective thermal conductivity and the experimental data of the pebble bed in SANA experiment under helium conditions. 
The calculated total effective thermal conductivity and the measured values in SANA experiment under nitrogen conditions are shown in Figure 7. As we can see, the total effective thermal conductivity under nitrogen conditions is a bit lower than that under helium conditions. However, the difference only comes from the second type of effective thermal conductivity due to different heat conduction properties of these two gases. The first and third type of effective thermal conductivities are the same under the two conditions, which are only related to the material and structure of the pebble bed.

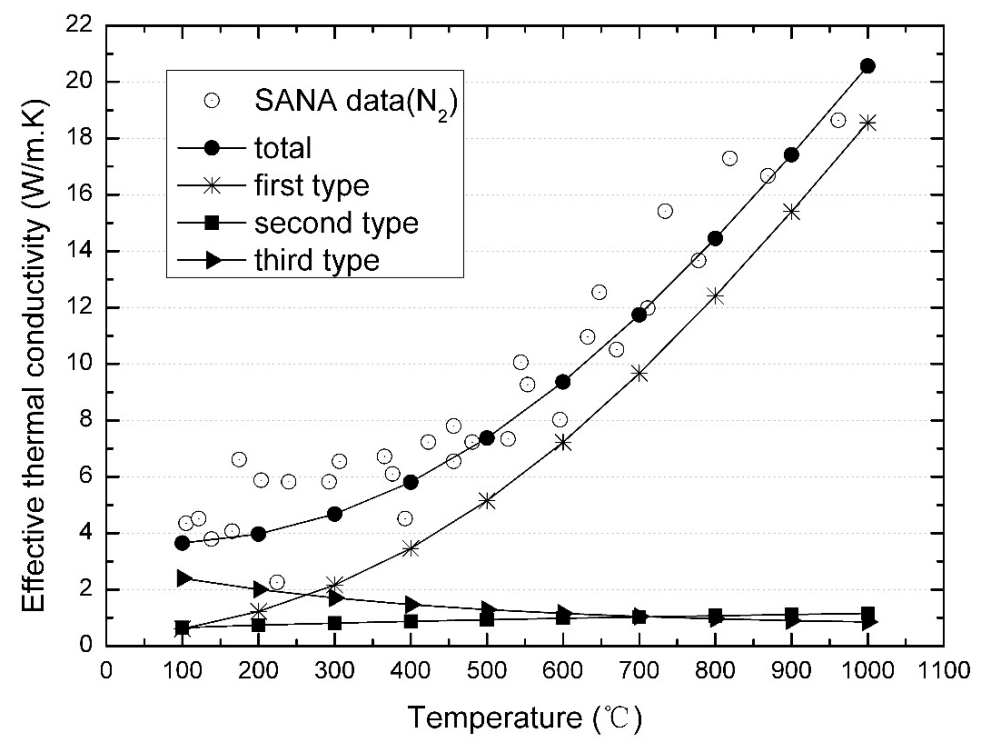

Figure 7. Total effective thermal conductivity and the experimental data of the pebble bed in SANA experiment under nitrogen conditions.

The TF-PBEC conducted by Tsinghua University aims to cover the whole temperature range of the safety analysis of HTR-PM $\left(0 \sim 1600{ }^{\circ} \mathrm{C}\right)$. Figure 8 gives the predicted total effective thermal conductivity of the pebble bed under helium conditions over the whole temperature range, which is useful for the design of insulating layers and heating power of the facility. Relevant parameters are used following the SANA experiment due to the similarity of the pebble bed.

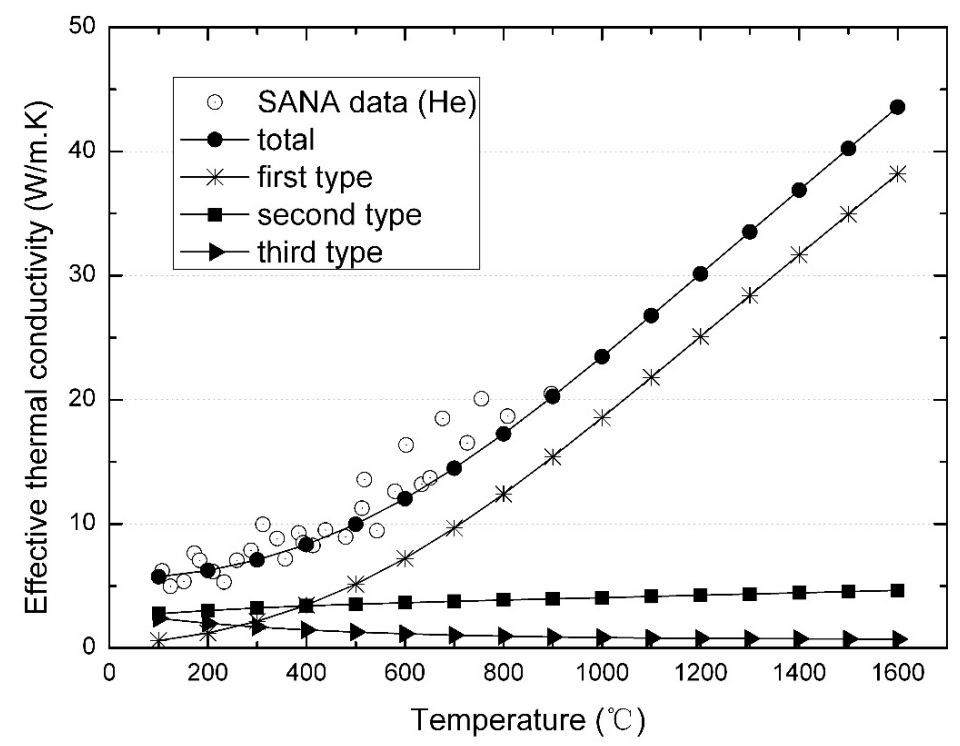

Figure 8. Total effective thermal conductivity of the pebble bed for helium conditions over the whole temperature range. 
However, it should be noted that we use a new fitting formula to describe the temperature-dependent heat conductivity of the pebble materials, as listed below.

$$
\lambda_{s}=141.10858 \times \mathrm{e}^{(-t / 382.46023)}+44.0461
$$

Figure 9 gives the comparison showing the old and new formula of the heat conductivity of the pebble material over the whole temperature range. As we can see in the picture, the star shows the experimental data of the heat conductivity of the pebble material. The circle shows the heat conductivity trend according to the recommended formula in the SANA report, while the inverted triangle shows the heat conductivity trend according to the new fitting formula. As we can see, the recommended quartic polynomial in the SANA report [12] gives a reasonable result below $1000{ }^{\circ} \mathrm{C}$. However, it gives an illogical result in the high temperature region.

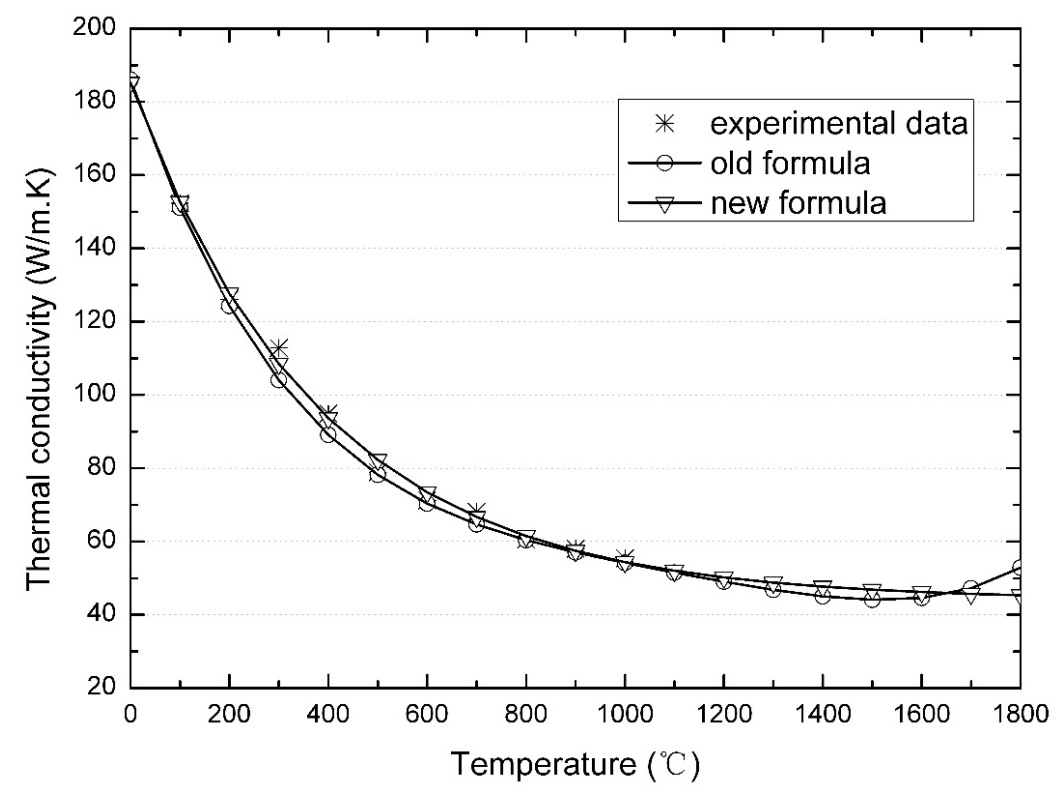

Figure 9. Comparison of the old and new fitting formula of the heat conductivity of the pebble material.

\section{Conclusions}

Pebble beds, which are formed by the random packing of a large number of particles, have broad applications in systems involving heat transfer $[18,19]$. Due to its complex microstructure, heat transfer through pebble bed contains several different mechanisms.

The effective thermal conductivity is used to represent the macroscopic heat transfer ability of the pebble bed. Three different types of effective thermal conductivity have been theoretically evaluated for the large-scale heat transfer test facility built for the HTR-PM. Results show that heat transfer by radiation plays a dominant role in the high temperature region, while heat transfer through gas conduction and contact area conduction occupies a major portion in the lower temperature region. The first and third type of effective thermal conductivities are only related to the pebble bed itself and the summation of the two can be considered as the effective thermal conductivity of the pebble bed under vacuum conditions. The prediction of the total effective thermal conductivity of the pebble bed over the whole temperature range is provided for the optimization of the test facility and guidance of further experiments.

Acknowledgments: The authors acknowledge the support of the National Natural Science Foundation of China under Grant No. 51506114, the Tsinghua University Initiative Scientific Research Program under Grant No. 2014z21023, the National S\&T Major Project of China under Grant No. ZX06901 and the Science Fund for Creative Research Groups of National Natural Science Foundation of China under Grant No. 51321002. 
Author Contributions: Cheng Ren and Xingtuan Yang contributed to the conception of the study and performed the data analyses. Haijun Jia helped perform the analysis and contributed to manuscript preparation. Yueyuan Jiang reviewed and edited the manuscript. Wei Xiong helped reviewed the manuscript and did constructive discussions. All authors read and approved the manuscript.

Conflicts of Interest: The authors declare that there is no conflict of interests regarding the publication of this paper.

\section{Acronym}

HTR high temperature pebble bed reactor

HTR-PM high temperature gas-cooled reactor pebble bed module

INET Institute of Nuclear and New Energy Technology

TF-PBEC test facility for pebble bed equivalent conductivity measurement

\section{References}

1. Yang, X.T.; Li, Y.; Gui, N.; Jia, X.L.; Tu, J.Y.; Jiang, S.Y. Some movement mechanisms and characteristics in pebble bed reactor. Sci. Technol. Nucl. Install. 2014, 2014, 820481. [CrossRef]

2. Jia, X.L.; Yang, X.T.; Gui, N.; Li, Y.; Tu, J.Y.; Jiang, S.Y. Experimental and Numerical Study of Stagnant Zones in Pebble Bed. Sci. Technol. Nucl. Install. 2014, 2014, 120640. [CrossRef]

3. Zhang, Z.; Wu, Z.; Wang, D.; Xu, Y.H.; Sun, Y.L.; Li, F.; Dong, Y.J. Current status and technical description of Chinese $2 \times 250$ MWth HTR-PM demonstration plant. Nucl. Eng. Des. 2009, 239, 1212-1219. [CrossRef]

4. Yang, X.T.; Sun, Y.F.; Ju, H.M.; Jiang, S.Y. Procedure of Active Residual Heat Removal after Emergency Shutdown of High-Temperature-Gas-Cooled Reactor. Sci. Technol. Nucl. Install. 2014, 2014, 583597. [CrossRef]

5. $\quad$ Ren, C.; Li, C.X.; Yang, X.T.; Liu, Z.Y.; Sun, Y.F.; Jiang, S.Y. Porous structure analysis of large-Scale randomly packed pebble bed in high temperature gas-cooled reactor. Atw-Int. J. Nucl. Power 2015, 60, 103-106.

6. Breitbach, G.; Barthels, H. The radiant heat transfer in the HTR Core after failure of the afterheat removal systems. Nucl. Technol. 1980, 49, 392-399.

7. Frano, R.L.; Aquaro, D.; Scaletti, L. Thermo-mechanical Characterization of Ceramic Pebbles for Breeding Blanket. Fusion Eng. Des. 2016, 109, 383-388. [CrossRef]

8. Frano, R.L.; Moscardini, M.; Aquaro, D. Numerical-experimental analyses by Hot-Wire method of an alumina cylinder for future studies on thermal conductivity of the fusion breeder materials. J. Phys. Conf. Ser. 2014, 547,1-9. [CrossRef]

9. Frano, R.L.; Aquaro, D.; Pupeschi, S.; Moscardini, M. Thermo-mechanical test rig for experimental evaluation of thermal conductivity of ceramic pebble beds. Fusion Eng. Des. 2014, 89, 1309-1313. [CrossRef]

10. Antwerpen, W.V.; Rousseau, P.G.; Toit, C.G.D. Multi-sphere Unit Cell model to calculate the effective thermal conductivity in packed pebble beds of mono-sized spheres. Nucl. Eng. Des. 2012, 247, 183-201. [CrossRef]

11. Antwerpen, W.V.; Toit, C.G.D.; Rousseau, P.G. A review of correlations to model the packing structure and effective thermal conductivity in packed beds of mono-sized spherical particles. Nucl. Eng. Des. 2010, 240, 1803-1818. [CrossRef]

12. Nießen, A.F.; Stocker, B. Sana-1 Code-to-experiment Summary Description of Benchmark. In Proceedings of the 3rd IAEA Research Coordination Meeting on Heat Transport and Afterheat Removal for Gas-Cooled Reactors under Accident Conditions, Vienna, Austria, 14-17 November 1995.

13. Ren, C.; Yang, X.T.; Li, C.X.; Liu, Z.Y.; Jiang, S.Y. Design of the material performance test apparatus for high temperature gas-cooled reactor. Nucl. Sci. Tech. 2013, 24, 060602.

14. Ren, C.; Yang, X.T.; Jiang, S.Y. Development of chinese HTR-PM pebble bed equivalent conductivity test facility. Atw-Int. J. Nucl. Power 2016, 61, 23-27.

15. Currie, J.A. Gaseous diffusion in porous media, Part 1. A non-steady state method; Part 2. Dry granular materials. Br. J. Appl. Phys. 1960, 11, 314-317, 318-324. [CrossRef]

16. Prasad, V.; Kladas, N.; Bandyopadhaya, A.; Tian, Q. Evaluation of correlations for stagnant thermal conductivity of liquid-saturated porous beds of spheres. Int. J. Heat Mass Trans. 1989, 32, 1783-1796. [CrossRef]

17. Kaviany, M. Principles of Heat Transfer in Porous Media; Springer-Verlag: New York, NY, USA, 1991; pp. 126-127. 
18. Aquaro, D.; Zaccari, N. Experimental and numerical analysis on pebble beds used in an ITER Test Module Blanket. Fusion Eng. Des. 2006, 81, 707-712. [CrossRef]

19. Antwerpen, W.V. Modelling the Effective Thermal Conductivity in the Near-Wall Region of a Packed Pebble Bed. Ph.D. Thesis, North-West University, Potchefstroom, South Africa, 2009.

(C) 2017 by the authors; licensee MDPI, Basel, Switzerland. This article is an open access article distributed under the terms and conditions of the Creative Commons Attribution (CC-BY) license (http://creativecommons.org/licenses/by/4.0/). 